\title{
A novel avalanche-confinement TEPC for microdosimetry at nanometric level
}

\author{
D. Bortot ${ }^{1,2^{*}}$, S. Agosteo ${ }^{1,2}$, P. Colautti ${ }^{3}$, V. Conte ${ }^{3}$, M.V. Introini ${ }^{1}$, M. Lorenzoli ${ }^{1}$, S. Pasquato ${ }^{1}$, A. Pola ${ }^{1,2}$ \\ ${ }^{1}$ Politecnico di Milano, Dipartimento di Energia, via La Masa 34, Milano, Italy \\ ${ }^{2}$ Istituto Nazionale di Fisica Nucleare - INFN, Sezione di Milano, via Celoria 16, Milano, Italy \\ ${ }^{3}$ Laboratori Nazionali di Legnaro LNL-INFN, viale dell'Università 2, Legnaro (Padova), Italy \\ *Corresponding Author: davide.bortot@polimi.it
}

\begin{abstract}
The tissue equivalent proportional counter (TEPC) is the most accurate device for measuring the microdosimetric properties of a particle beam, showing to properly assess the relative biological effectiveness by linking the physical parameters of the radiation with the corresponding biological response. Nevertheless no detailed information on the track structure of the impinging particles can be obtained, since the lower operation limit of the common TEPCs is about $0.3 \mu \mathrm{m}$. On the other hand, the pattern of particle interactions at the nanometer level, which demonstrated to have a strong correlation with radiation-induced damages to the DNA, is directly measured by only three different nanodosimeters worldwide: practical instruments are not yet available.

The gap between microdosimetry and track-nanodosimetry can be filled partially by extending the TEPC response down to the nanometric region. A feasibility study of a novel TEPC designed to simulate biological sites in the nanometric domain was performed. The present paper aims at describing the design, the development and the characterization of this avalanche-confinement TEPC. Irradiations with photons, fast neutrons and low-energy carbon ions demonstrated the capability of this TEPC of measuring in the range $0.3 \mu \mathrm{m}-25 \mathrm{~nm}$.
\end{abstract}

\section{KEYWORDS: microdosimetry, nanodosimetry, tissue equivalent proportional counter (TEPC), carbon ions}

\section{Introduction}

In the last three decades a great interest has grown for hadron therapy, which consists in a radiation therapy modality based on charged particles (protons and other ions such as carbon ions) for treating radio-resistant cancers and malignant tumors close to critical organs ${ }^{[1]}$. At present, 43 proton and 8 carbon ion therapy facilities are in operation worldwide, and many other are being planned or constructed $^{[2]}$. The assessment of the biological effective dose of clinical hadron beams is based on the measurements of the absorbed dose, which is a macroscopic quantity that demonstrated to be not adequate to describe the energy deposition process at micrometric level, because it does not take into account neither the stochastic of particle interaction in the target volume nor the track structure of ionizing charged particles, which is fundamental for initiating of the radiation damage ${ }^{[3]}$. A more comprehensive physical knowledge of the local energy deposition can be accomplished with high accuracy by innovative methodologies and instruments provided by microdosimetry, which aims at characterizing the statistical fluctuations of the local energy imparted at the micrometric level, and track-nanodosimetry, devoted to the description of the pattern of particle interactions at the nanometric level.

The tissue equivalent proportional counter (TEPC) is the most accurate device to measure the microdosimetric properties of a particle beam, and it is capable of simulating site sizes in the micrometric domain. At present, transportable devices are available, allowing to characterize radiation fields. Nevertheless, since the lower operation limit of standard TEPCs is about $0.3 \mu \mathrm{m}$, no detailed information on the track structure can be obtained.

On the other hand, the pattern of particle interactions at the nanometer level is measured by track-nanodosimetry, which derives the single-event distribution of ionization cluster size for site dimensions from a few nanometers up to tens of nanometers. Nanodosimetric probability distributions have demonstrated to show a trend similar to the cellular inactivation cross-sections ${ }^{[4]}$. Anyway, only three detectors are available worldwide for measuring these distributions. In order to fill the gap between standard TEPCs and nanodosimeters, an avalanche-confinement TEPC capable of simulating biological sites down to the nanometric region was designed and constructed. This detector was calibrated with an internal alpha source. Irradiations with photons emitted by a Cs-137 source demonstrated the good performances of the device and the reproducibility of the calibration procedure. Finally its response to fast neutrons and to low-energy carbon ions was assessed experimentally. 


\section{Methods}

\section{Design and development of the counter}

A TEPC can simulate site sizes in the nanometric domain only if the electronic avalanche is confined in a defined region inside the sensitive volume in order to guarantee a proper energy resolution of the device.

For this reason, an innovative TEPC based on a three-electrodes structure and capable of performing microdosimetric measurements from $0.3 \mu \mathrm{m}$ down to $25 \mathrm{~nm}$ in simulated site size, was designed basing on a prototype ${ }^{[5]}$. The peculiarities of the new design are mainly a thinner wall chamber, which allows the microdosimeter to measure low-energy (and high-LET) hadron beams. Moreover, a removable internal alpha source and a very compact solid state detector were inserted inside the sensitive zone for energy calibration. The detector sensitive volume is cylindrical, $13 \mathrm{~mm}$ in diameter and length and houses three electrodes independently biased: a central anode wire (graphite, $1 \mathrm{~mm}$ in diameter), a cylindrical cathode shell (conductive plastic A-150 type, $13 \mathrm{~mm}$ in internal diameter and $1 \mathrm{~mm}$ in thickness) and a helix (gold-plated tungsten, $100 \mu \mathrm{m}$ in diameter) made of 19 coils, $6 \mathrm{~mm}$ in diameter, which surrounds the anode wire and subdivides the sensitive volume into a drift and a multiplication region. Two field tubes (stainless steel, $6 \mathrm{~mm}$ in diameter) are employed for both sustaining the helix and defining the sensitive volume, thus avoiding any distortion of the electric field, while two insulating Rexolite caps enclose the chamber. An orifice on the basis cap permits the gas inlet and outlet (Figure 1).

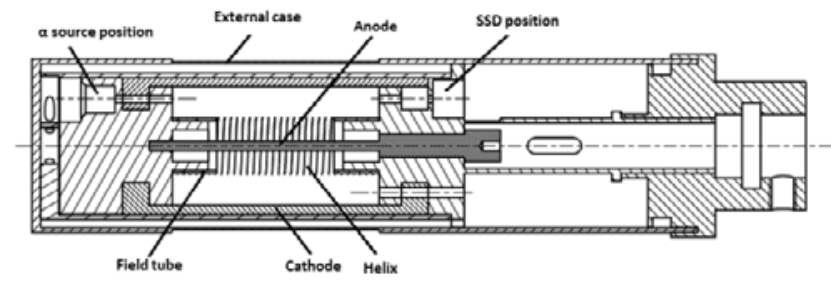

Figure 1: Cross sectional view of the new avalanche-confinement TEPC.

Two aligned holes were also designed in order to contain a thick removable $\mathrm{Cm}-244$ alpha source, sealed by a mylar layer, and a miniaturized solid state detector (SSD). This configuration allows to determine the response of the TEPC as a function of i) the simulated site size and ii) the polarization of the three electrodes. It guarantees that only signals due to alpha particles with a straight path inside the sensitive volume, i.e. the drift region, are collected.

Figure 2 shows the main components of the microdosimeter before its assembly and the assembled TEPC without the external case: the SSD cables and the small white hose for the gas inlet can be recognized.

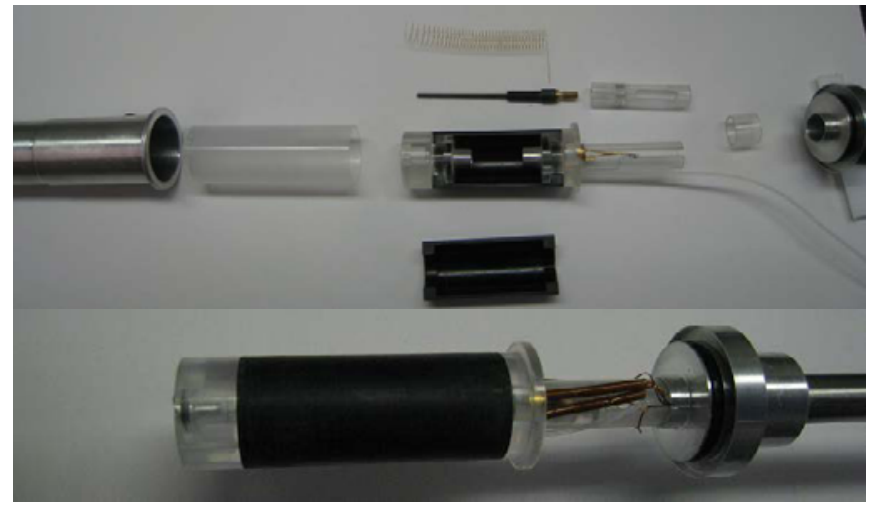

Figure 2: Pictures of the components which compose the TEPC and of the assembled device (without the external case).

\section{Vacuum and gas flow system}

A customized and transportable vacuum and gas flow system was developed in order to guarantee vacuum conditions and ensure a continuous replacement of tissue equivalent gas inside the chamber ${ }^{[6]}$. In such a way, the high purity required for stable gas gain during the irradiation is preserved and the ageing and outgassing effects are minimized.

Dimethyl ether (DME: $\left.\left(\mathrm{CH}_{3}\right)_{2} \mathrm{O}\right)$ is the selected filling gas for this avalanche-confinement TEPC: it can be considered as a tissue-equivalent gas, apart from the lack of nitrogen ${ }^{[7]}$. With respect to other tissue-equivalent gases, DME was chosen for its high gain (that leads to a better signal to noise ratio), its low diffusion coefficient (which increases the transparency of the helix to the drifted electrons), the presence of the chemical bond between carbon and oxygen (which lowers the polymerization rate, which is the aging effect of the anode wire) and its compatibility with all the materials of the TEPC.

\section{Operating conditions and energy calibration}

The characterization was devoted to optimize the operating conditions of the detection system by determining the proper bias voltage and pressure combinations, the stability and the performances of the whole system. This was accomplished through the determination of the so-called "electron transmission windows", empirical charts reporting the optimum operating conditions of the device in terms of polarization of the three electrodes at a selected simulated site size. A procedure for the calibration of the microdosimetric spectra based on the internal alpha source was also set up and exploited.

Irradiations with photons emitted by a Cs-137 isotopic source confirmed that the tested operating parameters of the device, i.e. the bias voltages of the electrodes which regulate the multiplication process, do not affect the microdosimetric information measured by the detector, which depends on the cavity size and on the radiation field only. 


\section{Applications}

The response of the novel TEPC to fast neutrons and to low-energy carbon ions was experimentally evaluated.

\section{Description}

Irradiations with a high-energy neutron field produced at the $\mathrm{CN}$ Van de Graaff accelerator of the INFN-Laboratori Nazionali di Legnaro (LNL-INFN) were performed. Fast neutrons up to $20 \mathrm{MeV}$ were generated through the ${ }^{7} \mathrm{Li}(\mathrm{d}, \mathrm{n})^{8} \mathrm{Be}$ reaction with $5.5 \mathrm{MeV}$ deuterons striking a thick Lithium target. This experimental characterization was carried out for simulated site sizes in the range $300 \mathrm{~nm}-25$ $\mathrm{nm}$ at different bias voltages.

Moreover, the response of this novel avalanche-confinement TEPC to a $62 \mathrm{AMeV}$ carbon ion beam was assessed at the carbon ion facility of the INFN-Laboratori Nazionali del Sud (LNS-INFN) in Catania. Several configurations were tested by varying both the simulated site size and the depth along the Bragg peak of the dose distribution of the delivered beam.

\section{Results with high energy neutrons}

Figure 3 (on the left) shows a neutron microdosimetric spectrum measured at a simulated site of $300 \mathrm{~nm}$. Three contributions can be observed: at low lineal energy values, ionization events are primarily due to electrons produced by gamma rays, while the central peak is mainly given by proton recoils produced through neutron elastic scattering. At higher $y$-values, the events are due to low-energy recoils (mainly carbon, nitrogen and oxygen ions) from the A-150 cathode wall. A contribution is also given by the ${ }^{4} \mathrm{He}$ ions produced by the neutron capture reaction on carbon.

Figure 3 (on the right) shows neutron spectra measured at $300,100,50,35,30$ and $25 \mathrm{~nm}$. Because of different values in the minimum detectable lineal energy, the spectra were normalized in order to obtain equal integrals for lineal

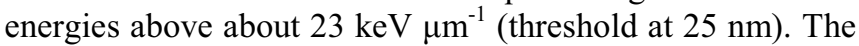
main proton peak at 300,100 and $50 \mathrm{~nm}$ is slightly shifted at higher lineal energies with respect to the other simulated sites. The distributions at 35,30 and $25 \mathrm{~nm}$ show a very similar shape, but different acquisition thresholds due to different values of the respective gain.
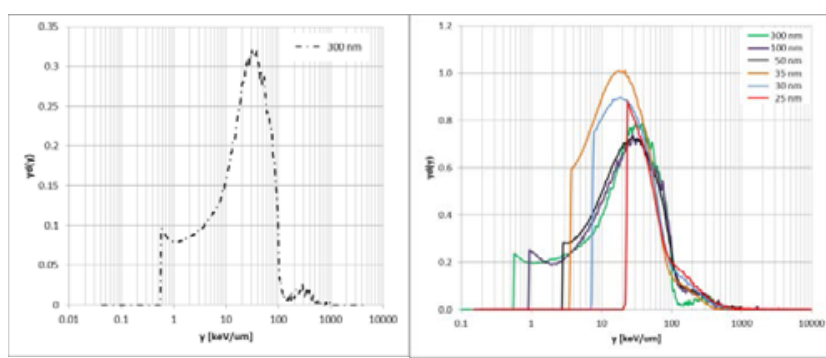

Figure 3: Neutron microdosimetric spectrum at $300 \mathrm{~nm}$ (left) and comparison between all measured neutron distributions at different simulated site sizes (right).
At $30 \mathrm{~nm}$ the main proton peak is still visible, even if located just above the threshold, while at $25 \mathrm{~nm}$ the limit condition is reached and only a small portion of the proton peak and of the contribution of light ions are present.

The reason of the discrepancy in the proton edge of the distributions measured at 300, 100 and $50 \mathrm{~nm}$ with respect to those derived at lower simulated sites is still under investigation. A future work will be devoted to irradiations with monoenergetic neutron fields in order to compare directly the experimental results with Monte Carlo simulations.

\section{Results with $62 \mathrm{AMeV}$ carbon ions}

A set of PMMA foils was placed in front of the detector in order to reproduce different depths across the Bragg peak.

Figure 4 shows the microdosimetric distributions measured at different simulated site sizes at the reference position, which corresponds to a total depth of $6.76 \mathrm{~mm}$ PMMA equivalent (in the distal part of the Bragg peak).

This comparison allows to appreciate a small change in the shape of carbon peak when the site size reduces from 300 $\mathrm{nm}$ to $100 \mathrm{~nm}$. Owing to different minimum detectable lineal energy, spectra at 50, 35 and $25 \mathrm{~nm}$ were normalized in order to obtain equal integrals for lineal energies above about $23 \mathrm{keV} \mathrm{\mu m}^{-1}$ (threshold at $25 \mathrm{~nm}$ ).

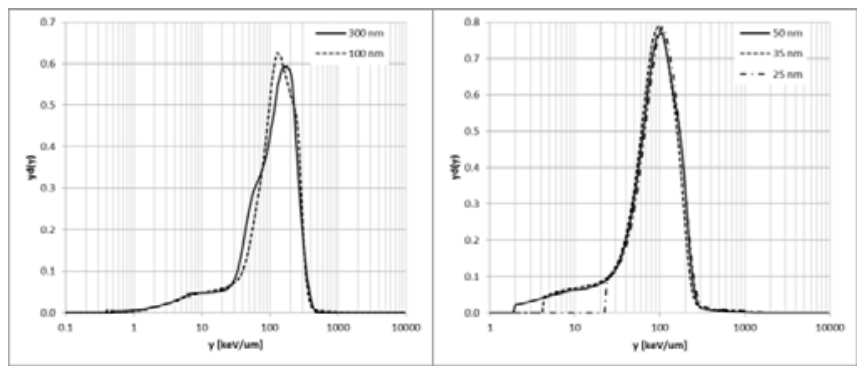

Figure 4: Spectra obtained by irradiating the TEPC at the reference depth $(6.76 \mathrm{~mm})$ with a $62 \mathrm{AMeV}$ carbon ion beam at $300 \mathrm{~nm}$ and $100 \mathrm{~nm}$ (left) and $50 \mathrm{~nm}, 35 \mathrm{~nm}$ and $25 \mathrm{~nm}$ (right).

Figure 5 (left) shows the comparison between the lineal energy spectra obtained for a simulated site of $300 \mathrm{~nm}$ at a depth $6.50 \mathrm{~mm}, 6.76 \mathrm{~mm}$ (reference point) and $7.09 \mathrm{~mm}$ in PMMA. At $6.50 \mathrm{~mm}$ the carbon peak is slightly shifted at lower lineal energies with respect to the one at $6.76 \mathrm{~mm}$, but the edge is the same. At $7.03 \mathrm{~mm}$, the spectrum changes completely: the primary carbon contribution is no more present and that of high energy fragments, which is barely visible at $6.50 \mathrm{~mm}$ and $6.76 \mathrm{~mm}$, becomes predominant. This depth is beyond the Bragg peak.

The microdosimetric spectra obtained at eight different points along the depth dose profile for a simulated site size of $25 \mathrm{~nm}$ are shown in the right part of Figure 5. The distributions shift towards higher y values as depth increases (from $1.76 \mathrm{~mm}$ to $6.76 \mathrm{~mm}$ ), according to the beam slowing-down. In contrast, at $7.29 \mathrm{~mm}$ in depth, which is beyond the Bragg peak, the distribution changes dramatically due to the contribution of high energy fragments. 


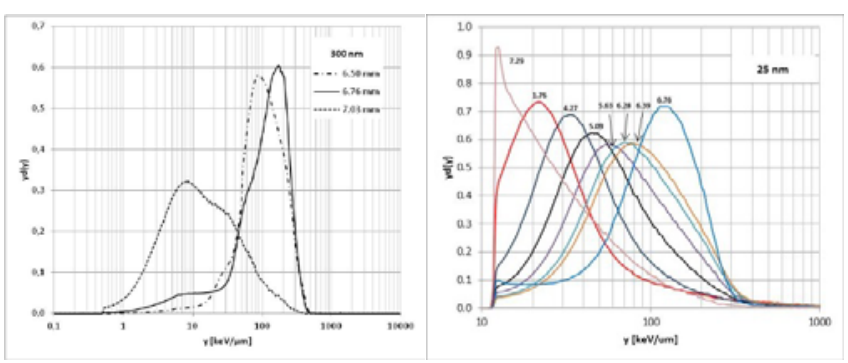

Figure 5: microdosimetric spectra obtained for a simulated site of (left) $300 \mathrm{~nm}$ at a depth 6.50, 6.76 and $7.03 \mathrm{~mm}$ in PMMA and (right) $25 \mathrm{~nm}$ at eight depths from $1.76 \mathrm{~mm}$ to $7.29 \mathrm{~mm}$.

\section{Conclusion}

The novel avalanche-confinement TEPC showed to be capable of measuring in the range $0.3 \mu \mathrm{m}-25 \mathrm{~nm}$ when irradiated with low-energy carbon ions. It should be stressed that microdosimetric measurements of hadron beams at the nanometric scale with portable devices have never been performed before.

A direct and systematic comparison with reference devices both in the micrometric and nanometric domains has to be performed and will be matter of future work. Moreover, a new facility constituted by a track-nanodosimeter coupled with a wall-less avalanche-confinement TEPC will be designed and constructed, for correlating directly the microdosimetric spectra with the track-nanodosimetric distributions at the same experimental conditions. The microdosimetric spectra measured by such a device consist in the convolution of core and penumbra events, the two components of a particle track measured through a nanodosimeter. A systematic comparison between the microdosimetric and track-nanodosimetric distributions for the same particle beam will allow to investigate the possibility of unfolding the microdosimetric spectrum into its different track components. In such a way, the beam characteristics measured by a portable TEPC could be transferred to a track-nanodosimetric description, much more relevant in the framework of the biological damages to the nanometric targets, such as the DNA and the chromosome fiber.

\section{Acknowledgment}

This work has been supported by the MITRA project (Scientific Commission V) of the Italian National Institute for Nuclear Physics-INFN.

\section{References}

1) M. Durante, J.S. Loeffler, Charged particles in radiation oncology, Nature Reviews Clinical Oncology 7: 37-43 (2010).

2) Particle Therapy Co-operative Group PTCOG: http://www.ptcog.ch/index.php/facilities-in-operation.
3) K. Weyrather, G. Kraft, $R B E$ of carbon ions: Experimental data and the strategy of RBE calculation for treatment planning, Radiotherapy and Oncology 73 (2): S161-S169 (2004).

4) V. Conte, D. Moro, P. Colautti, B. Grosswendt, Nanodosimetric descriptors of the radiation quality of carbon ions, Radiation Protection Dosimetry 166 (1-4): 214-218 (2015).

5) V. Cesari et al., Nanodosimetric measurements with an avalanche confinement TEPC, Radiation Protection Dosimetry 99(1-4): 337-342 (2002).

6) D. Bortot et al., Development of a Vacuum and Gas Flow System for a New Avalanche Confinement TEPC, INFN Website, LNL Annual Report 2013.

7) International Commission on Radiation Units and Measurement (ICRU), Microdosimetry, Report 36 (1983). 